\title{
Diagnostic accuracy of postmortem computed tomography for bleeding source determination in cases with hemoperitoneum
}

\author{
Vasiliki Chatzaraki $^{1,2}$ (D) Michael J. Thali ${ }^{1} \cdot$ Garyfalia Ampanozi $^{1}$ (D)
}

Received: 22 July 2020 / Accepted: 18 November 2020 / Published online: 7 January 2021

(C) The Author(s) 2021

\begin{abstract}
Aim The aim of this retrospective study was to determine the accuracy of postmortem computed tomography and different radiological signs for the determination of the bleeding source in cases with hemoperitoneum confirmed at autopsy.

Methods Postmortem computed tomography data of consecutive cases with hemoperitoneum confirmed at autopsy were reviewed by two raters, blinded to the autopsy findings. The determination of possible bleeding sources was based on the presence of the sentinel clot sign, blood or sedimented blood surrounding an organ, intraparenchymal abnormal gas distribution, and parenchymal disruption. The bleeding source and the cause of hemoperitoneum (traumatic, surgical, natural, or resuscitation) as reported in the autopsy report were noted. The survival intervals of the deceased were calculated when information about the time of an incident related to death was available in the autopsy reports.

Results Eighty-five cases were included in the study. Postmortem computed tomography showed $79 \%$ sensitivity and $92.1 \%$ specificity for the detection of the bleeding source. The sentinel clot sign was associated with surgical or natural causes of hemoperitoneum and longer survival intervals. Sedimented blood around the bleeding source was associated with resuscitation. Abnormal gas distribution within organs and combination of multiple radiological signs provided higher sensitivity.

Conclusion Postmortem computed tomography provides moderate sensitivity and high specificity for determining the bleeding source in cases with hemoperitoneum. Different PMCT signs are associated with different causes of hemoperitoneum and survival intervals.
\end{abstract}

Keywords Hemoperitoneum $\cdot$ Abdominal hemorrhage $\cdot$ Bleeding source $\cdot$ Forensic radiology $\cdot$ Autopsy $\cdot$ Postmortem computed tomography (PMCT)

Key points

1. PMCT shows moderate sensitivity and high specificity for abdominal bleeding sources' detection.

2. Sedimented blood surrounding the bleeding source is associated with resuscitation.

3. The sentinel clot sign is associated with surgical or natural causes and longer survival intervals.

4. Intraparenchymal abnormal gas distribution and combination of PMCT signs provide high sensitivity.

Vasiliki Chatzaraki

chatzaraki@outlook.com.gr

1 Department of Forensic Medicine and Imaging, Institute of Forensic Medicine, University of Zurich, Winterthurerstrasse 190/52, CH-8057 Zurich, Switzerland

2 Department of Radiology, Kantonsspital Baden AG, Im Ergel 1, CH-5404 Baden, Switzerland

\section{Introduction}

Hemoperitoneum can be traumatic, spontaneous, or iatrogenic. Traumatic hemoperitoneum can be the result of solid organ, vasculature, bowel, mesenteric, or bladder injuries with the spleen and liver being the most frequently injured organs by blunt force and penetrating trauma [1-3]. Spontaneous hemoperitoneum has visceral organ-, gynecologic-, vascular-, and coagulopathy-related causes [4]. Iatrogenic causes include any surgical procedure performed in the intraperitoneal cavity usually combined with postoperative anticoagulation therapy [4].

In the clinical setting, non-contrasted computed tomography (CT) comprises an important screening tool for trauma management [5] and provides high sensitivity for the detection of small intraperitoneal effusions [1]. CT also provides high specificity and negative predictive value for the decision-making process regarding whether a liver injury requires intervention [6]. Radiological findings related to hemoperitoneum are 
intraperitoneal blood accumulation [1], the sentinel clot sign (high-attenuation blood clot adherent to an injured organ) [1, 7, $8]$, and directly visible organ injuries (parenchymal inhomogeneities, contusions, lacerations, hematomas) [1].

Blood usually has higher density than other intraperitoneal fluids (around 30-45 Hounsfield units (HU)) [1]. The sentinel clot sign is an indirect clue for determining the bleeding source in cases of hemoperitoneum $[1,4,9,10]$. It is characterized by a focal heterogeneous area of clotted blood, which is denser than the rest of the fluid in the abdomen, indicating the site of the organ or vessel injured [7, 8]. The mean density of clotted blood is approximately within the range of 45-70 HU.

In the forensic routine, unenhanced postmortem CT (PMCT) is increasingly used to supplement autopsy. Postmortem computed tomography angiography (PMCTA) provides high sensitivity for the detection of essential findings regarding vessels and organ lesions; however, it is more time-consuming and therefore is mainly applied as a targeted examination in the forensic routine $[11,12]$. Compared to antemortem CT, PMCTA, and autopsy, PMCT shows a more limited efficacy for diagnosing or ruling out abdominal injuries and particularly small ones [13, 14]. However, the sensitivity of PMCT for determination of hemoperitoneum is high [15], and the more life-threatening an abdominal injury, the higher the sensitivity [16]. Typical radiological findings of hemoperitoneum can be observed also postmortem, like blood surrounding an injured organ [15], sentinel clot $[17,18]$, parenchymal inhomogeneity, and abnormal gas distribution not following the vascular tree within solid organs [15]. Blood appears different on PMCT compared to antemortem imaging because of the postmortem changes and blood sedimentation $[19,20]$.

The primary aim of this study was to determine the diagnostic accuracy of PMCT and specific radiological signs for the determination of the intra-abdominal bleeding source in cases with hemoperitoneum confirmed at autopsy. Secondary aim was to examine PMCT accuracy for the detection of the bleeding source with regard to raters' confidence for the radiological diagnosis, the cause of bleeding, and the survival interval.

\section{Materials and methods}

\section{Subjects}

All consecutive cases with age $\geq 18$ years and postmortem interval $\leq 4$ days based on the estimation of time of death [21-24] that underwent pre-autopsy PMCT and full forensic autopsy from January 1, 2011, to December 31, 2018, were retrospectively reviewed in our database. Autopsy reports were reviewed for recorded presence of hemoperitoneum. In cases with reported hemoperitoneum, the reports were further assessed for the bleeding source. Only cases with hemoperitoneum and the bleeding source at autopsy clearly found to be at least one of the following organs were included in the study: liver, spleen, right kidney, left kidney, pancreas, right adrenal gland, left adrenal gland, any abdominal artery. Cases with blood exclusively in the retroperitoneal space and not in the peritoneal cavity at autopsy were not included in this study. Organ findings at autopsy not considered as possible bleeding source, for example subcapsular hematomas of the liver, were not considered as relevant for this study. Exclusion criteria contained the following: (1) cases with other than pure blood accumulation in the abdomen, (2) not clearly stated bleeding source in the autopsy report, (3) cases with bowel and/or mesenterial rupture as bleeding site, (4) cases with intraperitoneal foreign bodies (including knives, projectiles, medical gauzes), (5) cases with gunshot, sharp force and fire injuries of the abdomen, and (6) destructing trauma with open injury of the peritoneum and/or abdominal organs' dislocations.

During the autopsy reports' review, the organs comprising bleeding sources were noted for every case. Additionally, the cause of bleeding was noted for every bleeding source as among traumatic, surgical/natural, or caused by resuscitation efforts. For the cases with traumatic and surgical/natural causes, the survival interval between the related incident and death was calculated, when applicable, based on the information given. The cases were divided into two categories of survival interval: $\leq 1 \mathrm{~h}$ and $>1 \mathrm{~h}$.

\section{Imaging protocol - PMCT review}

PMCT was performed on a 128-slice scanner (SOMATOM Definition Flash, Siemens Healthineers, Erlangen, Germany), with the bodies in supine position, using automatic dose modulation (CARE Dose 4D ${ }^{\mathrm{TM}}$, Siemens Healthineers, Erlangen, Germany). Imaging parameters were as follows [25]: tube voltage $120 \mathrm{kV}$, slice collimation $128 \times 0.6 \mathrm{~mm}$. Image reconstructions of the abdomen were performed [25], with a slice thickness of $1.0 \mathrm{~mm}$ and an increment of $0.6 \mathrm{~mm}$. The arms were elevated if possible, in order to avoid artifacts.

Two raters (a forensic pathology trainee with 2.5 years' experience and a board-certified forensic pathologist with 11 years' experience in forensic radiology) evaluated the PMCT data in consensus reading for the radiological determination of the bleeding source based on the presence of the following five radiological signs: (1) sentinel clot sign (highattenuation irregular fluid accumulation/blood clot around an organ or vessel while the rest fluid in the abdomen provides lower attenuation values); (2) hyperdense fluid (blood) around the organ (homogeneous fluid accumulation without internal density deviations); (3) sedimented blood around the organ (fluid-fluid level); (4) abnormal gas distribution within solid organs not following the vascular tree; and (5) parenchymal disruptions/lacerations. The raters were blinded to the autopsy findings during PMCT review. After the radiological 
determination of the possible bleeding source, the raters rated the confidence of every diagnosis based on a 3-point confidence-level Likert scale (level $1=$ the organ could be bleeding source, level $2=$ the organ is probably bleeding source, level $3=$ the organ is definitely a bleeding source).

\section{Bleeding source determination based on PMCT signs}

The sensitivity (SEN), specificity (SPC), positive predictive (PPV), negative predictive value (NPV), and positive and negative likelihood ratios of PMCT for the detection of the bleeding source were calculated independently of organ, as well as organ-dependently. Autopsy was considered the reference test.

The proportions of the true positive (TP) and false positive (FP) signs were calculated with respect to the distinct PMCT signs.

For the TP bleeding sources on PMCT, the relationships between survival interval and PMCT sign and between cause of the bleeding and PMCT sign were investigated.

\section{Raters' confidence for detection of bleeding sources on PMCT}

The confidence levels given by the raters were assessed with regard to the distinct PMCT signs. The relationship of the three confidence levels with the TP or FP diagnosis was investigated independently of the different radiological signs.

\section{Radiological signs with regard to cause of intra- abdominal bleeding}

The distribution of the TP bleeding sources on PMCT in the three different cause of bleeding groups (trauma/surgical-natural/resuscitation) was examined. The frequencies of the radiological signs were compared among the different cause of bleeding groups.

\section{Survival interval with regard to performance of PMCT and radiological signs}

For the cases with traumatic and surgical/natural causes of bleeding and available information in the autopsy reports, the survival interval was calculated and groups of $\leq 1 \mathrm{~h}$ and $>1 \mathrm{~h}$ were created. For the positive organs on PMCT, by which bleeding was caused by the abovementioned causes (and not by resuscitation), the proportions of TP and FP findings were compared between the two survival interval groups.

For the TP findings, the proportions of the different radiological signs were compared between the two different survival interval groups.

\section{Statistical analysis}

Statistical analysis was performed with the SPSS Statistical Package (@ IBM, SPSS 20, Chicago, IL, USA). Chi-square tests and Fischer's exact tests of independence were used to compare proportions of nominal variables between nominal variables' groups. $P$ values less than 0.05 indicated statistical significance. Excel (Microsoft Excel, (C) 2010 Microsoft Corporation, USA) was used for creating graphs.

\section{Results}

\section{Subjects}

One hundred thirty-six cases with hemoperitoneum at autopsy were identified in the database. Fifty-one were excluded; thus, 85 cases were included in the study. Sixty-three (74\%) concerned males (age $49 \pm 19$ years) and $22(26 \%)$ females (age $59 \pm 16$ years). From the 85 cadavers included in the study, 57 $(67 \%)$ were scanned with the arms elevated during PMCT; in the rest 28 (33\%), elevating the arms was avoided because of extended external injuries or postmortem rigidity.

From the 679 assessed organs $(85$ cases $\times 8$ organs $/$ case $=$ $680-1$ case of absent left kidney=679), 124 were determined as bleeding sources at autopsy (livers 60 , spleens 40 , right kidneys 4 , left kidneys 6 , pancreas 0 , right adrenal glands 2 , left adrenal glands 1, vessels 11: abdominal aorta 7, splenic artery 1 , renal artery 1 , external iliac artery 1 , femoral artery 1). For $104(84 \%)$ of the 124 bleeding sites, the cause was traumatic, for $12(10 \%)$ surgical or natural, and the rest $8(6 \%)$ were caused by resuscitation measures. Calculation of the survival interval of the subjects was applicable for 92 out of the 124 bleeding sites. Seventy-nine bleeding sites were associated with survival interval $\leq 1 \mathrm{~h}$ and 13 to $>1 \mathrm{~h}$.

\section{Bleeding source determination based on PMCT signs}

The overall performance of PMCT for the detection of the bleeding source found at autopsy organ-dependently, as well as independently of organ, is presented in Table 1 . From the 124 bleeding sites, 26 were missed by PMCT (false negatives). Overall SEN of PMCT for the detection of an abdominal bleeding source was 79\% (95\% CI 70.8 to 85.8 ) (Table 2). From the 555 organs without pathological findings at autopsy, PMCT was positive for 44 (FP) (Table 1). Overall SPC was 92.1\% (95\% CI 89.5 to 94.2 ) (Table 2).

From the 142 positives on PMCT ( $98 \mathrm{TP}+44 \mathrm{FP}$ ), 39 were considered as positive because of present sentinel clot sign, 54 because of blood collection surrounding the related organ, 13 because of sedimented blood around the organ, 23 because of intraparenchymal abnormal gas distribution, and 11 based on combinations of signs (Table 3). 
Table 1 Organ-based and organ-independent (total) $2 \times 2$ table evaluating the number of the organs determined as positive or negative bleeding source on PMCT with regard to autopsy results

\begin{tabular}{|c|c|c|c|c|}
\hline & & Autopsy + & Autopsy - & Total \\
\hline \multirow[t]{3}{*}{ Liver } & $\mathrm{PMCT}+$ & 47 & 3 & 50 \\
\hline & PMCT - & 13 & 22 & 35 \\
\hline & Total & 60 & 25 & 85 \\
\hline \multirow[t]{3}{*}{ Spleen } & $\mathrm{PMCT}+$ & 31 & 22 & 53 \\
\hline & PMCT - & 9 & 23 & 32 \\
\hline & Total & 40 & 45 & 85 \\
\hline \multirow[t]{3}{*}{ Kidney right } & $\mathrm{PMCT}+$ & 4 & 10 & 14 \\
\hline & PMCT - & 0 & 71 & 71 \\
\hline & Total & 4 & 81 & 85 \\
\hline \multirow[t]{3}{*}{ Kidney left } & $\mathrm{PMCT}+$ & 5 & 4 & 9 \\
\hline & PMCT - & 1 & 74 & 75 \\
\hline & Total & 6 & 78 & 84 \\
\hline \multirow[t]{3}{*}{ Pancreas } & $\mathrm{PMCT}+$ & 0 & 4 & 4 \\
\hline & PMCT - & 0 & 81 & 81 \\
\hline & Total & 0 & 85 & 85 \\
\hline \multirow[t]{3}{*}{ Adrenal gland right } & $\mathrm{PMCT}+$ & 1 & 0 & 1 \\
\hline & PMCT - & 1 & 83 & 84 \\
\hline & Total & 2 & 83 & 85 \\
\hline \multirow[t]{3}{*}{ Adrenal gland left } & $\mathrm{PMCT}+$ & 0 & 0 & 0 \\
\hline & PMCT - & 1 & 84 & 85 \\
\hline & Total & 1 & 84 & 85 \\
\hline \multirow[t]{3}{*}{ Vessel } & $\mathrm{PMCT}+$ & 10 & 1 & 11 \\
\hline & PMCT - & 1 & 73 & 74 \\
\hline & Total & 11 & 74 & 85 \\
\hline \multirow[t]{3}{*}{ Total } & $\mathrm{PMCT}+$ & 98 & 44 & 142 \\
\hline & PMCT - & 26 & 511 & 537 \\
\hline & Total & 124 & 555 & 679 \\
\hline
\end{tabular}

$P M C T+$, organs with at least one radiological sign on PMCT; PMCT - , organs without radiological signs on PMCT; $A U T+$, organs positive at autopsy; $A U T-$, organs intact at autopsy

In each organ group, 85 organs were evaluated (one organ/case) except of the left kidney where in one case it was absent

\section{Sentinel clot sign}

A total of $66.7 \%$ of the organs determined positive on PMCT based on the presence of the sentinel clot sign were TP (Table 3, Fig. 1, Fig. 2, Fig. 3, Fig. 4).

From the $44 \mathrm{FP}$ in total, 13 (4 right kidneys, 1 pancreas, 7 spleens, and 1 left renal artery) were incorrectly determined positive based on the sentinel clot sign: sentinel clot was detected for the right kidney in one case of abdominal aortic rupture (Fig. 3), in one spleen laceration, in one liver laceration (but the fatty capsule of the right kidney exhibited hemorrhages at the autopsy), and in one case with liver and right adrenal gland injuries. FP sentinel clot was also determined for the pancreas in a case of spleen artery aneurysm rupture (Fig. 3). Interestingly, FP sentinel clot sign for the spleen was detected in 6 cases where actually the liver was the single organ injured. In another case, FP sentinel clot sign for spleen was determined where actually a splenic artery aneurysm rupture was the source. In a further case, sentinel clot sign was determined for the renal artery on PMCT; however, a spleen laceration was the actual bleeding site.

\section{Blood or sedimented blood around the organ}

Twenty-one organs were FP based on the presence of surrounding blood and 6 based on the presence of surrounding sedimented blood (Fig. 1, Fig. 2). Two livers were considered as positive (one because of blood and one because of sedimented blood), whereas the spleen was actually the source in both cases (Fig. 3). Twelve spleens were FP where actually only the liver (in the 11 cases) or the liver and the right kidney (in one case) were bleeding. Interestingly, from the 4 cases with FP, sedimented blood surrounding the spleen, in 3 the liver, was the only source as result of resuscitation. Ten kidneys (6 right and 4 left) were FP based on presence of surrounding blood and blood in the retroperitoneal space, whereas only the liver (in 5 cases) or the liver and the spleen (in 5 cases) were actually bleeding bleeding (Fig. 5). In 3 cases, the pancreas was FP, whereas in the 2 of them, both the liver and spleen were injured and in one splenic aneurysm rupture was the source (Fig. 3).

\section{Abnormal gas distribution and parenchymal disruption}

A total of $91.3 \%$ of the organs with abnormal gas distribution on PMCT were TP (Fig. 1, Fig. 2). A total of $8.7 \%(n=2)$ of them were FP, concerning 2 spleens with paradoxically suspected abnormal gas distribution on PMCT (confidence level for both spleens was equal to 3), whereas in another one, the liver and in another one the left kidney were injured. Regarding parenchymal disruption, only 2 organs showed parenchymal disruption as a single sign of possible injury on PMCT; the one (spleen) was TP but the other was FP (liver with confidence level $=3$, where only the spleen was injured).

\section{Combination of signs indicating bleeding source}

Eleven organs showed a combination of signs on PMCT indicating bleeding source and all were TP (Fig. 1, Fig. 2, Fig. 4). Five showed the combination of sentinel clot with parenchymal disruption, 4 sentinel clot with abnormal gas distribution, 1 abnormal gas distribution with sedimented blood, and 1 abnormal gas distribution with parenchymal disruption.

\section{Raters' confidence for detection of bleeding sources on PMCT}

Confidence levels given by the raters with regard to the five different radiological signs are presented in Fig. 6. Overall, 
Table 2 Sensitivity $(S E N)$, specificity $(S P C)$, positive and negative predictive value ( $P V V$ and $N P V)$, and positive (+) and negative (-) likelihood ratios of PMCT for the detection of the bleeding source in cases with hemoperitoneum independently of organ (row at the bottom-total) and organ-dependently

SEN (\%) (95\% CI) SPC (\%) (95\% CI) PPV (\%) (95\% CI) NPV (\%) (95\% CI) + Likelihood ratio - Likelihood ratio $(95 \% \mathrm{CI}) \quad(95 \% \mathrm{CI})$

\begin{tabular}{lllllll}
\hline Liver & $78.3(65.8,87.9)$ & $88(68.8,97.5)$ & $94(84.3,97.9)$ & $62.9(50.6,73.7)$ & $6.53(2.24,19.03)$ & $0.25(0.15,0.41)$ \\
Spleen & $77.5(61.6,89.2)$ & $51.1(35.8,66.3)$ & $58.5(50,66.5)$ & $71.9(57.4,82.9)$ & $1.59(1.13,2.23)$ & $0.44(0.23,0.84)$ \\
Kidney right & $100(39.8,100)$ & $87.7(78.5,93.9)$ & $28.6(18.3,41.7)$ & $100(-)$ & $8.1(4.5,14.5)$ & 0 \\
Kidney left & $83.3(35.9,99.6)$ & $94.9(87.5,98.6)$ & $55.6(31.1,77.6)$ & $98.7(92.5,99.8)$ & $16.3(5.9,45)$ & $0.18(0.03,1.05)$ \\
Kidney (right + left) & $90(55.5,99.75)$ & $91.2(85.7,95.1)$ & $39.1(27.2,52.5)$ & $99.3(95.8,99.9)$ & $10.2(5.9,17.6)$ & $0.11(0.02,0.7)$ \\
Pancreas & - & $95.3(88.4,98.7)$ & - & - & - & 1.05 \\
Adrenal gland right & $50(1.3,98.7)$ & $100(95.7,100)$ & 100 & $98.8(95.4,99.7)$ & - & $0.5(0.13,2)$ \\
Adrenal gland left & $0(0,97.5)$ & $100(95.7,100)$ & - & $98.8(93.6,99.9)$ & - & $1(1,1)$ \\
Adrenal gland (right + left) & $33.3(0.8,90.6)$ & $100(97.8,100)$ & 100 & $98.8(97.4,99.5)$ & - & $0.67(0.3,1.5)$ \\
Vessel & $90.9(58.7,99.8)$ & $98.7(92.7,99.8)$ & $90.9(58.6,98.6)$ & $98.7(91.9,99.8)$ & $67.3(9.5,475.5)$ & $0.09(0.01,0.6)$ \\
Total & $79(70.8,85.8)$ & $92.1(89.5,94.2)$ & $69(62.3,75)$ & $95.2(93.3,96.5)$ & $10(7.4,13.5)$ & $0.23(0.16,0.32)$ \\
\hline
\end{tabular}

independently of radiological sign, higher confidence levels were not associated to higher proportions of TP findings (chisquare test; $p=0.22$ ).

\section{Radiological signs with regard to cause of intra- abdominal bleeding}

From the 99 TP bleeding sources, 82 (82.8\%) were result of trauma, $11(11.1 \%)$ of a surgical or natural cause, and $6(6.1 \%)$ of resuscitation measures. The distribution of the radiological signs was different among the cause of bleeding groups (Fischer's exact test; $p=0.001$, Fig. 7). TP bleeding sources that were caused by surgical or natural causes appeared most usually with the sentinel clot sign ( $81.8 \%$ within surgical/natural causes), whereas half (50\%) of the TP bleeding sources caused by resuscitation appeared with surrounding sedimented blood. TP bleeding sources caused by trauma mainly appeared with blood in the region $(37.8 \%)$, abnormal gas distribution (25.6\%), sentinel clot (19.5\%), and combination of signs (11\%) (Fig. 7).

\section{Survival interval with regard to performance of PMCT and radiological signs}

For the positive organs on PMCT, by which the bleeding was caused by trauma, surgery, or natural causes (and not resuscitation), the proportions of TP and FP findings were not different between the two survival interval groups (TP 75\% in $\leq 1 \mathrm{~h}$ and $62.5 \%$ in $>1 \mathrm{~h}$ interval, FP $25 \%$ in $\leq 1 \mathrm{~h}$ and $37.5 \%$ in $>$ $1 \mathrm{~h}$ interval, chi-square test; $p=0.36$ ).

For the TP findings $(n=99)$, by which the bleeding was caused by trauma, surgical, or natural causes $(n=$ 76), the distribution of the different radiological signs was significantly different between the survival interval groups (Fischer's exact test; $p=0.009$, Fig. 7). The sentinel clot sign appeared significantly more often in longer survival intervals $(50 \%$ within $>1$ h vs $15.2 \%$ within $\leq 1 \mathrm{~h}$ group). The same phenomenon was observed for sedimented blood, however with not so prominent differences. On the other hand, blood surrounding the bleeding source was a sign which appeared more often in shorter intervals ( $\leq 1 \mathrm{~h} 40.9 \%$ vs $>1 \mathrm{~h} 10 \%$ ). Abnormal gas distribution and parenchymal disruptions were
Table 3 The distribution of the organs with positive radiological sign/signs on PMCT in the true positive $(\mathrm{TP}=\mathrm{PMCT}+$, AUT +$)$ and false positive (FP $=$ PMCT + , AUT-) groups with regard to the different radiological signs

\begin{tabular}{lllr}
\hline & TP & FP & \multicolumn{1}{c}{ Total } \\
\hline Sentinel clot $(n$ organs $-\%)$ & $26(66.7 \%)$ & $13(33.3 \%)$ & $39(100 \%)$ \\
Blood $(n$ organs $-\%)$ & $33(61.1)$ & $21(38.9)$ & $54(100 \%)$ \\
Sedimented blood $($ N organs $-\%)$ & $7(53.8 \%)$ & $6(46.2 \%)$ & $13(100 \%)$ \\
Gas distribution $(n$ organs - \%) & $21(91.3 \%)$ & $2(8.7 \%)$ & $23(100 \%)$ \\
Parenchymal disruption $(n$ organs $-\%)$ & $1(50 \%)$ & $1(50 \%)$ & $2(100 \%)$ \\
Combination $(n$ organs $-\%)$ & $11(100 \%)$ & 0 & $11(100 \%)$ \\
Total $(n$ organs $-\%)$ & $99(69.7 \%)$ & $43(30.3 \%)$ & $142(100 \%)$ \\
\hline
\end{tabular}


Fig. 1 Bar chart depicting the frequencies $(\%)$ of the organs determined as positive on PMCT, i.e., the organs with at least one radiological sign on PMCT ("PMCT + organs"; true positive with green and false positive with red) with regard to the different radiological signs

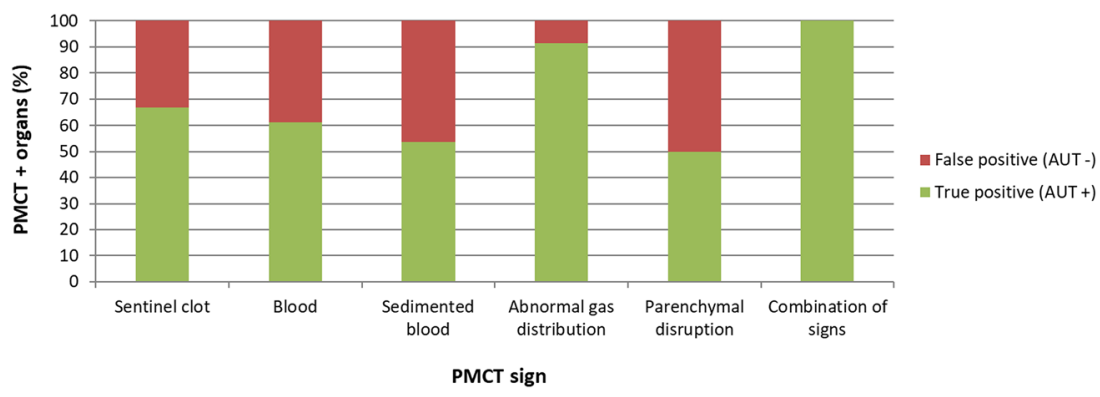

notably more in count in $\leq 1 \mathrm{~h}$ as single signs, as well as part in combination of signs, 6 cases with combination of signs in $\leq 1 \mathrm{~h}$ group, whereas 3 showed sentinel clot with parenchymal disruption, 2 sentinel clot with abnormal gas distribution, 1 parenchymal disruption with abnormal gas distribution, and 3 cases in $>1 \mathrm{~h}$ group, whereas the 2 showed sentinel clot with parenchymal disruption and 1 abnormal gas distribution with sedimented blood around.

\section{Discussion}

Apart from the overall diagnostic performance of PMCT, this study attempted to investigate the diagnostic performance of specific PMCT signs for the detection of the bleeding site in cases with hemoperitoneum. The presence of different signs was examined with regard to the cause of hemoperitoneum and survival interval. SEN was medium, while SPC was high for predicting the bleeding source. Different radiological signs are associated with different causes of hemoperitoneum and survival intervals.

The studied sample ( 85 cases with a total of 124 bleeding sites at autopsy) was relatively large compared to previous studies on PMCT $[15,16,26]$. The previous studies assessed PMCT accuracy for the detection of abdominal injury in road traffic accident victims [26] and traumatic deaths $[15,16]$. Though the current study examined PMCT accuracy for the bleeding site only in

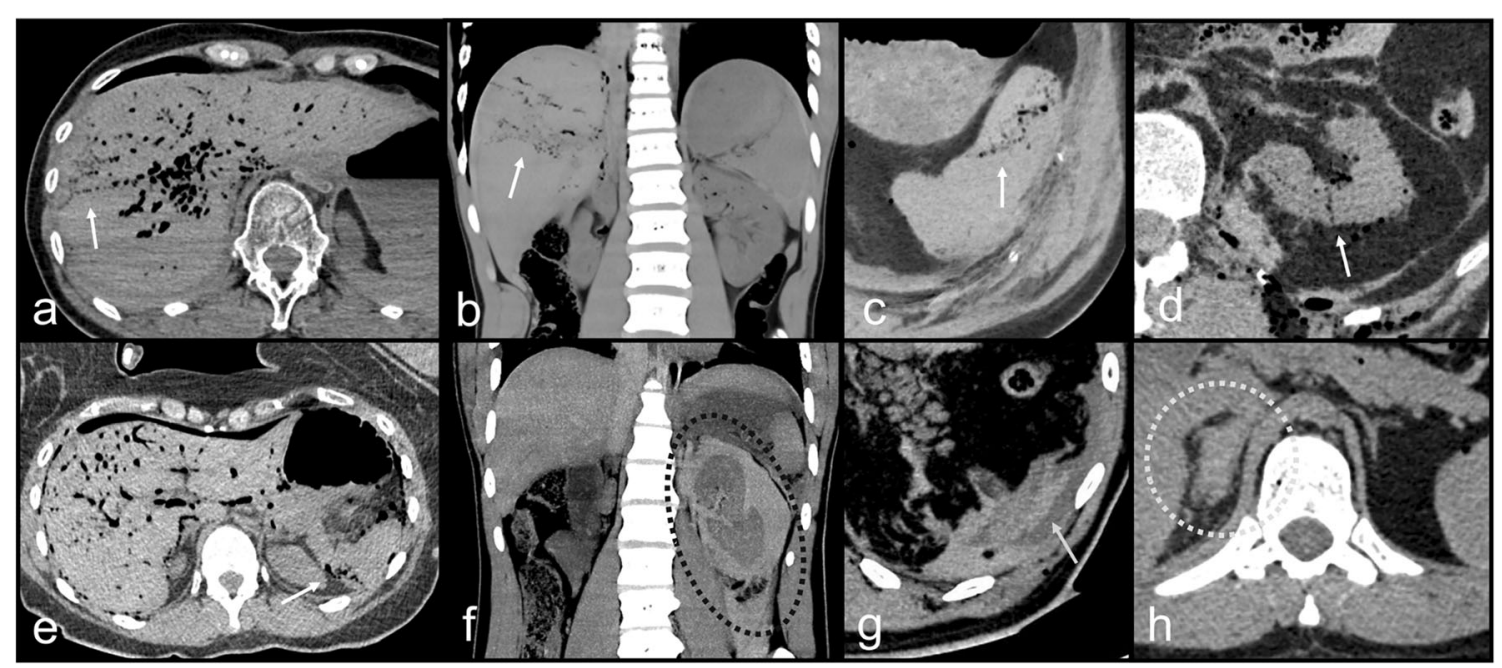

Fig. 2 PMCT multiplanar reconstructions (MPR) in different cases with true positive abnormal findings. a PMCT axial slice through the abdomen; true positive abnormal gas distribution not following the vascular tree within the liver. b PMCT coronal slice through the abdomen; true positive abnormal gas distribution within the liver not following the vascular tree. PMCT axial slices through the spleen (c) and left kidney (d); true positive abnormal gas distributions within a spleen and a kidney. e PMCT axial slice through the abdomen; true positive abnormal gas distribution within the spleen. Note the prominent but normally distributed gas in the liver within the vascular tree. Liver was true negative in this case. f PMCT coronal slice through the abdomen; extended parenchymal disruption of the left kidney with sentinel clot sign. g PMCT axial slice through the spleen; sentinel clot sign. h PMCT axial slice through the right adrenal gland; blood around the right adrenal gland (note the difference with the left one) as true positive sign on PMCT 


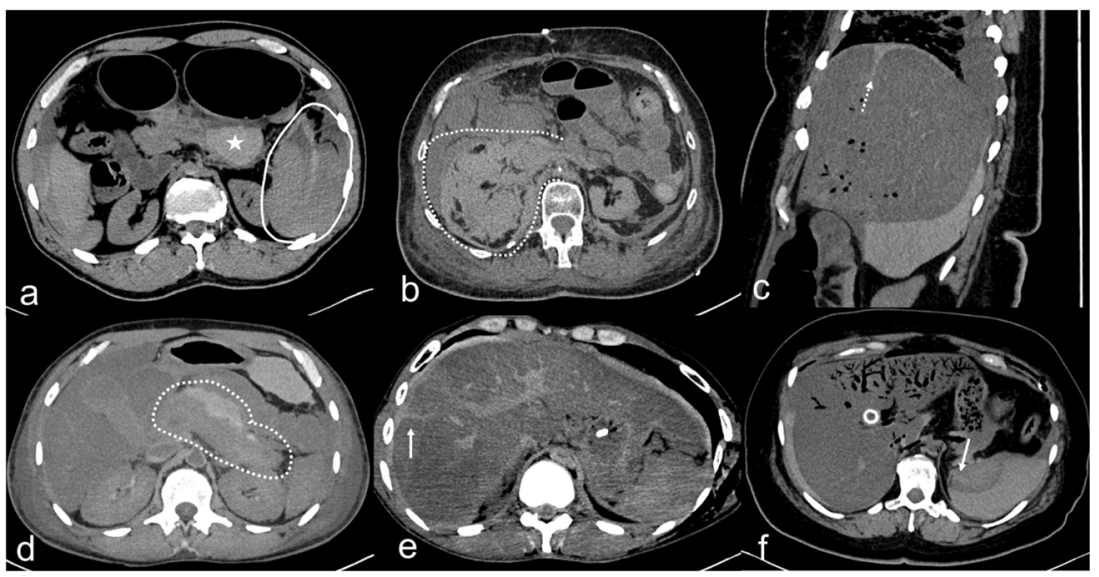

Fig. 3 PMCT multiplanar reconstructions (MPR) in different cases with false positive (a-d) and one case with true positive (e) abnormal findings. a PMCT axial slice through the abdomen; false positive sedimented blood around the pancreas (asterisk) in a case of spontaneous splenic artery rupture (white line). b PMCT axial slice through the abdomen; false positive sentinel clot for the right kidney in a case of aortic rupture. c PMCT sagittal slice through the abdomen of the same case with $\mathrm{f}$ (details in f). $\mathbf{d}$ PMCT axial slice through the abdomen; false positive sentinel clot sign around the pancreas in a case where actually the liver and the spleen were injured. e PMCT axial slice through the abdomen of a case with true positive parenchymal disruption of the right liver lobe and sentinel clot sign around the liver. $f$ PMCT-axial slice through the abdomen of a case with false positive parenchymal disruption of the liver (misinterpretation of a hepatic groove - dashed arrow in c). Note the true positive parenchymal disruption of the spleen (white arrow in $f$ ) with sedimented blood around the organ cases with hemoperitoneum diagnosed at autopsy, the total SEN and SPC (79\% and $92.1 \%$, respectively) were similar to those found by Alvarez et al. (79.6\% and $94.1 \%$, respectively) [15]. However, the organ-specific SEN and SPC values of the current study were on average higher than those presented by Christe et al. (liver on PMCT alone, SEN 53\%, SPC 84\%; spleen on PMCT and magnetic resonance imaging (PMMR), SEN 50\%, SPC 89\%; kidneys on PMCT and PMMR, SEN 25\%, SPC 100\%) probably because of their smaller sample and because the reported organ injuries in their study were small and therefore difficult to detect in imaging [16]. SEN and SPC for liver and spleen were similar with that found by Norzailin et al. [26] and slight higher for the liver, spleen, and kidneys than those found by Juźwik et al. [27]. In a previous study with larger sample, injuries of pancreas were considered rare with PMCT providing medium sensitivity (71.4\%) but high specificity (96.1\%). In the current study, no pancreas injuries were detected at autopsy [27].

A total of $67 \%$ of the bleeding sources determined positive on PMCT based on the sentinel clot sign were TP. FP sentinel clot was very frequent for the right kidney and the spleen while other organs actually comprised the bleeding sites (most usually the liver). The sentinel clot sign was associated with natural and surgical causes, as well as with longer survival intervals $(>1 \mathrm{~h})$. These observations may compound with the pathophysiological basis of the sign, which lies on the defending mechanism of the body against fatal blood loss outside the cardiovascular system. As hemostasis is attempted at the rupture site, a blood clot is formed, which provides a different radiological appearance than the lysed blood because of the different hemoglobin

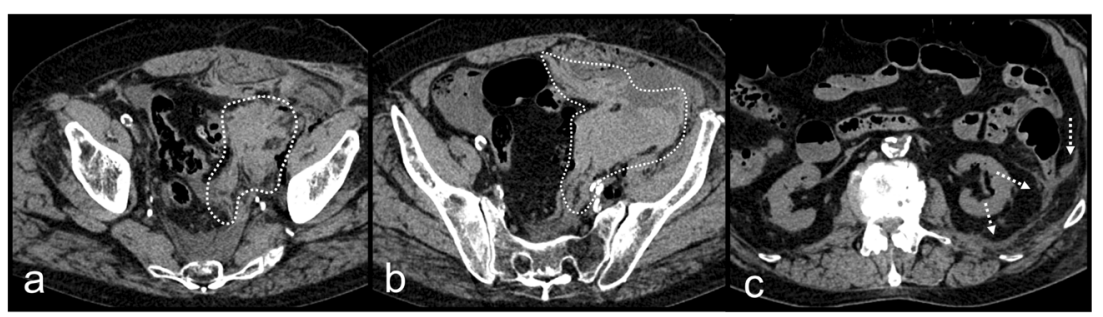

Fig. 4 PMCT of a case with true positive findings for acute rupture of the left common femoral artery PMCT axial slices through the pelvis $(\mathbf{a}, \mathbf{b})$ and the retroperitoneal spaces at renal level (c); true positive sentinel clot sign of the left femoral artery. Note the accumulation of hyperdence fluid at the level of the artery in the pelvis ( $\mathbf{a}$ and $\mathbf{b}$ ), whereas the expanded fluid in the retroperitoneal spaces (c) is visually more hypodense 


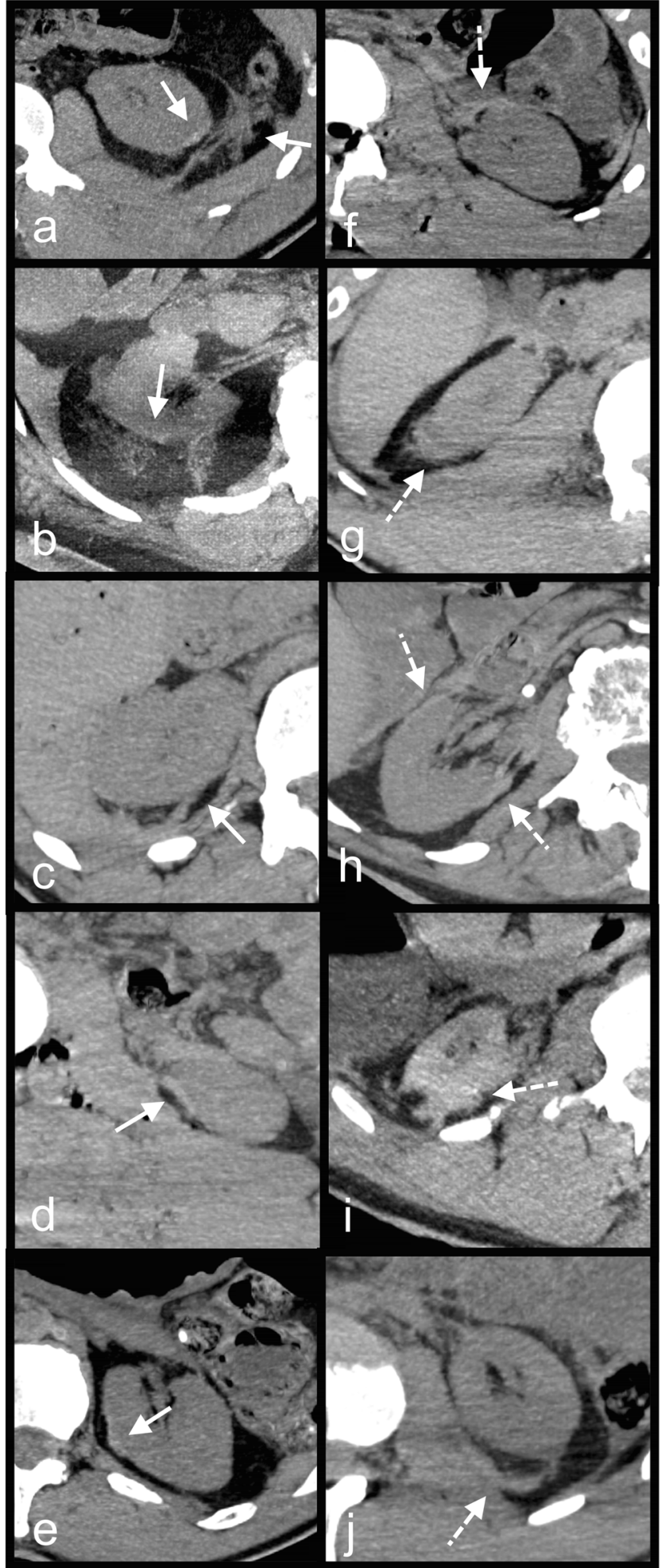

Fig. 5 a-e PMCT axial slices through true positive kidneys (true positive findings with white arrows). $\mathbf{f}-\mathbf{j}$ PMCT axial slices through false positive kidneys (false positive findings with white dashed arrows) levels and subsequently different radiodensities [7]. It is assumed that clot formation requires a certain time and very short survival intervals may temporally not allow this hemostatic process to be sufficiently developed.

Blood and sedimented blood surrounding the assumed bleeding site were also TP for approximately $60 \%$ of the positive organs on PMCT. FP blood and sedimented blood accumulations around the spleen and the kidneys were usual in cases where actually only the liver or the liver and the spleen (in cases of FP kidneys) were the bleeding site. Blood accumulation was associated with short survival intervals $(\leq 1 \mathrm{~h})$, whereas sedimented blood with larger ones $(>1 \mathrm{~h})$. This can be explained by the fact that the cellular blood components are allowed to sink and sediment within the vascular system and cavities (i.e., hemothoraces, hemoperitoneum) over time. This phenomenon may be not fully developed in very short survival intervals. Early sedimentation may be associated with longer agonal intervals ("In individuals dying a slow lingering death with terminal cardiac failure, livor mortis may actually appear antemortem" [24]). Interestingly, sedimented blood around the bleeding site was more usual in cases where bleeding was caused by resuscitation. This observation conforms to observations about hemopericardium's radiological appearance in cases with antemortem (associated with double-band sign) and cases with hemopericardium caused by postmortem manipulations and resuscitation (single-band sign or horizontal level) [28, 29].

It seems that in cases where clot is not clearly or not at all formed and subsequently invisible on PMCT, the blood floats and may be re-distributed in the abdominal cavity (i.e., from the right subphrenic to the left subphrenic space and vice versa or from the subphrenic spaces through the paracolic gutters into the pelvis). There exists communication between the retrohepatic space and the retroperitoneal perirenal space (so-called Kneeland channel) [30], which also allows the transportation of blood from the peritoneal to the retroperitoneal spaces, and this can explain the large number of FP kidney signs where actually only the liver or the liver and the spleen or the abdominal aorta was bleeding.

Abnormal intraparenchymal gas distribution not following the vascular tree was TP for $90 \%$ of the positive cases on PMCT and was associated with traumatic causes and short survival intervals $(\leq 1 \mathrm{~h})$. It is assumed that injuries appearing with abnormal gas distribution are related to more severe and life-threatening conditions 

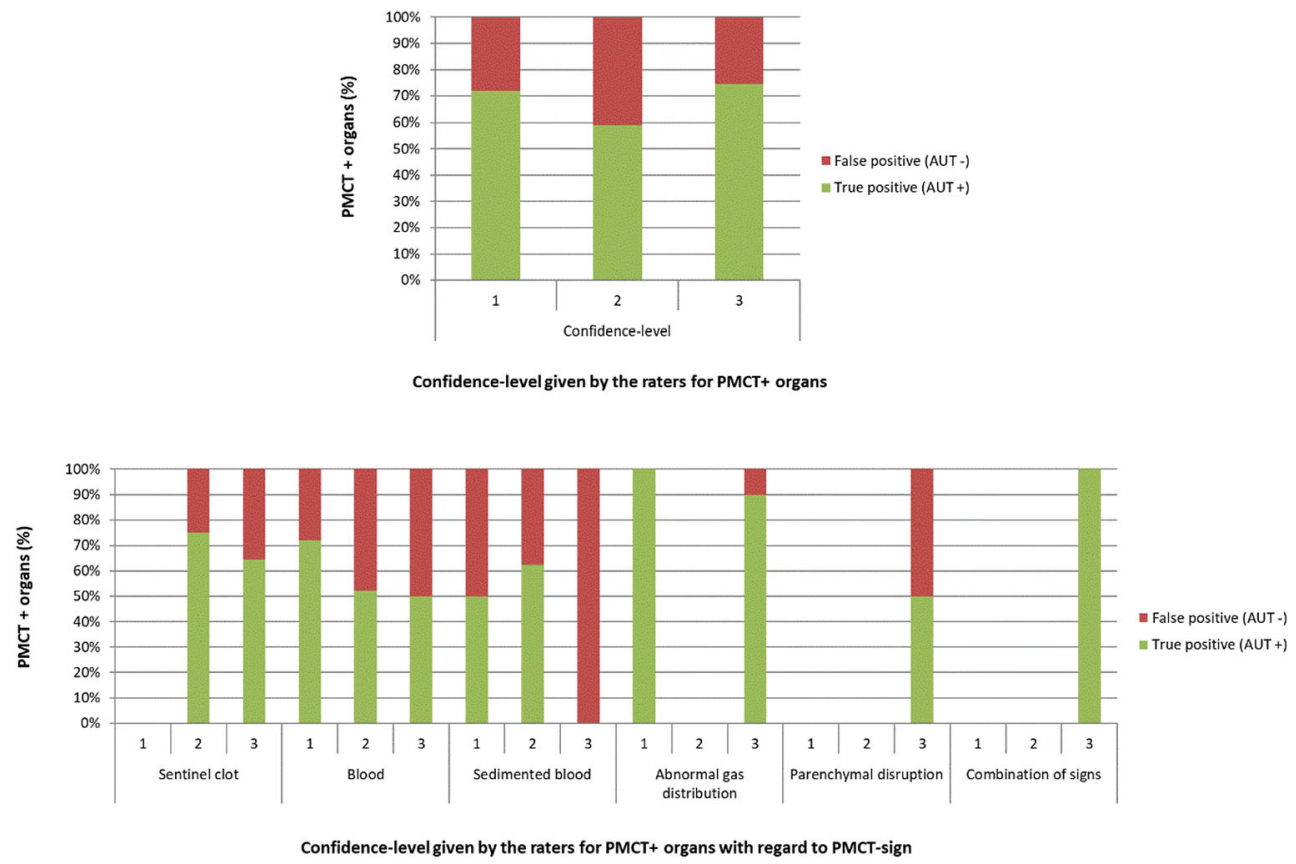

Fig. 6 Bar charts depicting the frequencies (\%) organs determined as positive on PMCT, i.e. the organs with at least one radiological sign on PMCT ("PMCT + organs"; true positive with green and false positive with red) with regard to the confidence level given by the raters (in consensus) when determined the organ as positive in total (chart on the top) and within the different PMCT signs (chart at the bottom). Higher confidence levels were not associated to higher proportions of true positive PMCT findings (chi-square test; $p=0.22$ ) and can subsequently be more easily visible on PMCT, increasing SEN [16]. The same was observed for parenchymal disruptions. It was reasonable to expect that combinations of radiological signs would increase the PMCT sensitivity for the detection of the bleeding source and indeed all organs determined as possible bleeding sites based on a combination of signs on PMCT were TP.

This study has limitations. Not all intraperitoneal organs were evaluated as possible bleeding sources. Parts of the gastrointestinal tract, the internal genital organs, gall, and urinary bladder were not assessed under the assumption that after their perforation, the consistency of the intraperitoneal fluid is not pure blood. Secondly, no pancreatic injuries were detected in this sample; thus, accuracy values for pancreas could not be extracted. Thirdly, although the raters were blinded to autopsy findings, detection bias may exist as they were aware that hemoperitoneum was present on all reviewed PMCT. However, the aim of the study was to determine the PMCT accuracy for the detection of the bleeding source and not for the detection of hemoperitoneum itself. Last but not the least, because of the retrospective manner of this study, a reliable extraction of information regarding possible medical handlings (infusions, transfusions, etc.) between incident and time of death was not possible based on the review of the autopsy reports, which may have brought bias in the estimation of the presented survival intervals and maybe also in the postmortem radiological appearance of hemoperitoneum. However, this condition may correspond to real conditions considering that such information often lacks in the forensic routine at the time a forensic radiologist is called to determine a possible bleeding source on PMCT.

\section{Conclusion}

PMCT provides moderate sensitivity and high specificity for the detection of abdominal bleeding sources in postmortem cases with hemoperitoneum. For the true positive organ findings, specific radiological signs may be associated to the different causes of bleeding and the survival interval.

Acknowledgments Open access funding provided by University of Zurich. The authors express their gratitude to Emma Louise Kessler, $\mathrm{MD}$, for her generous donation to the Zurich Institute of Forensic Medicine, University of Zurich, Switzerland. 
Fig. 73 Graph on the top. Bar chart depicting the frequencies (\%) of the PMCT signs of true positive organs (organs with at least one radiological sign on PMCT and positive at autopsy; "PMCT+, AUT+", $n=99$ ) with regard to the cause of bleeding (as determined at autopsy and based to case history). The distribution of the radiological signs was different among the cause of bleeding groups (Fischer's exact test; $p=0.001)$. Graph at the bottom. Bar chart depicting the frequencies (\%) of the PMCT signs of the true positive organs (organs with at least one radiological sign on $\mathrm{PMCT}$ and at autopsy: "PMCT+, AUT+", $n=$ 99) with cause of bleeding being trauma, operation or natural causes (and not resuscitation) ( $n=$ $76)$ in the two different survival interval groups $(\leq 1 \mathrm{~h}$ or $>1 \mathrm{~h})$. The distribution of the different PMCT signs was significantly different between the survival interval groups (Fischer's exact test; $p=0.009$ )
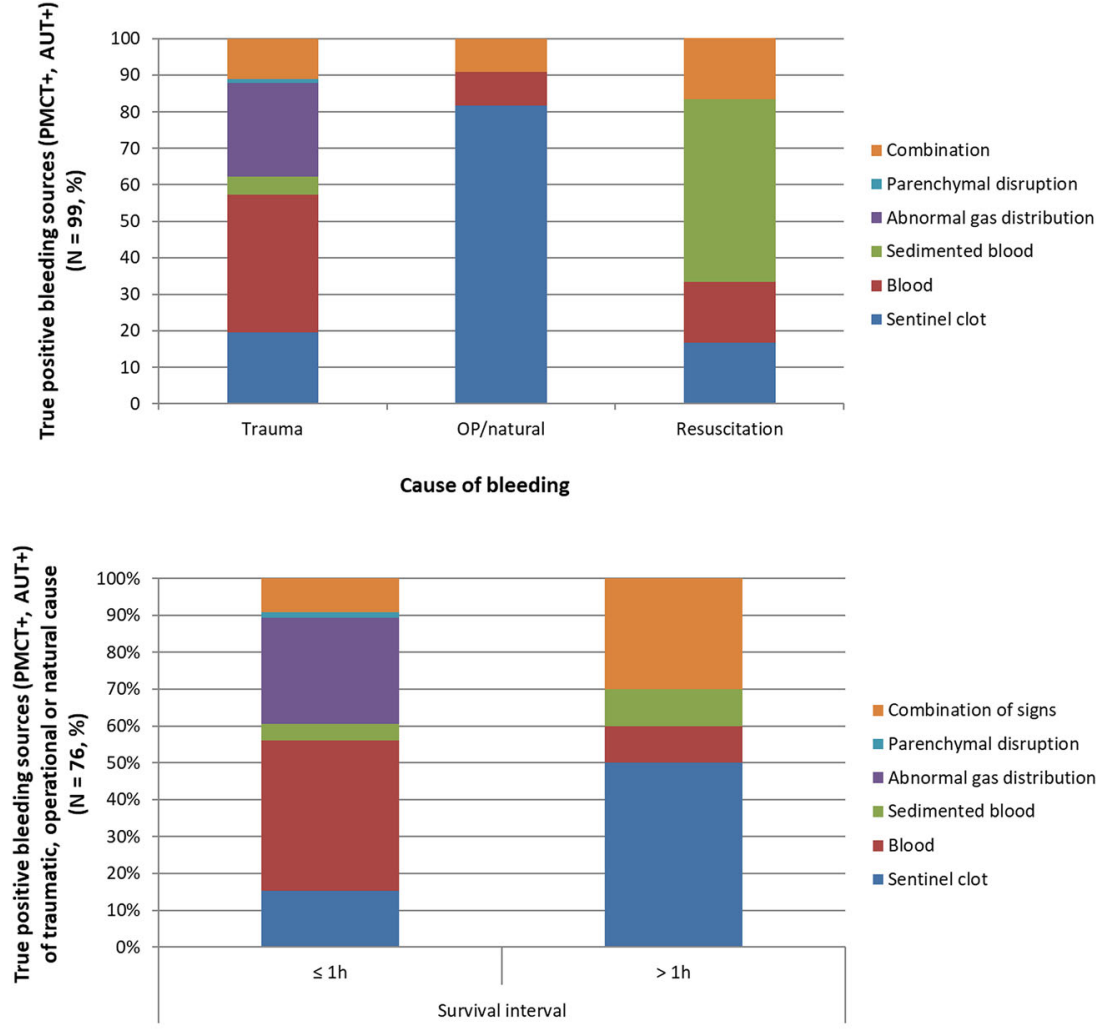

Survival interval groups

\section{Compliance with ethical standards}

Data use for this study conforms with the Swiss laws and ethical standards as approved by the Cantonal Ethics Committee of Zurich, Switzerland (KEK ZH-Nr. 2015-0686).

Conflict of interest The authors declare that they have no conflict of interest.

Ethical approval Ethical approval was obtained by the Cantonal Ethics Committee of Zurich, Switzerland, Nr. 2015-0686. This article does not contain any studies with animals performed by any of the authors.

Open Access This article is licensed under a Creative Commons Attribution 4.0 International License, which permits use, sharing, adaptation, distribution and reproduction in any medium or format, as long as you give appropriate credit to the original author(s) and the source, provide a link to the Creative Commons licence, and indicate if changes were made. The images or other third party material in this article are included in the article's Creative Commons licence, unless indicated otherwise in a credit line to the material. If material is not included in the article's Creative Commons licence and your intended use is not permitted by statutory regulation or exceeds the permitted use, you will need to obtain permission directly from the copyright holder. To view a copy of this licence, visit http://creativecommons.org/licenses/by/4.0/.

\section{References}

1. Lubner M, Menias C, Rucker C, Bhalla S, Peterson CM, Wang L, Gratz B (2007) Blood in the belly: CT findings of hemoperitoneum. RadioGraphics 27:109-125. https://doi.org/10.1148/rg.271065042

2. Shin SS, Jeong YY, Chung TW, Yoon W, Kang HK, Kang TW, Shin HY (2007) The sentinel clot sign: a useful CT finding for the evaluation of intraperitoneal bladder rupture following blunt trauma. Korean J Radiol 8:492-497. https://doi.org/10.3348/kjr.2007.8.6.492

3. Gudelj M, Giroul F, Dorthu L (2018) Intraperitoneal bladder rupture revealed by the sentinel clot sign. J Belgian Soc Radiol 102:12. https://doi.org/10.5334/jbsr.1517

4. Furlan A, Fakhran S, Federle MP (2009) Spontaneous abdominal hemorrhage: causes, CT findings, and clinical implications. Am J Roentgenol 193:1077-1087. https://doi.org/10.2214/AJR.08.2231

5. Hamilton JD, Kumaravel M, Censullo ML, Cohen AM, Kievlan DS, West OC (2008) Multidetector CT evaluation of active extravasation in blunt abdominal and pelvic trauma patients. RadioGraphics 28 : 1603-1616. https://doi.org/10.1148/rg.286085522

6. MacLean AA, Durso A, Cohn SM et al (2005) A clinically relevant liver injury grading system by $\mathrm{CT}$, preliminary report. Emerg Radiol 12:34-37. https://doi.org/10.1007/s10140-005-0441-0

7. Orwig D, Federle M (1989) Localized clotted blood as evidence of visceral trauma on CT: the sentinel clot sign. Am J Roentgenol 153: 747-749. https://doi.org/10.2214/ajr.153.4.747 
8. Yudin A (2014) Metaphorical signs in computed tomography of chest and abdomen. Springer, Berlin, pg. 67, Sentinel Clot Sign

9. Swensen S, McLeod R, Stephens D (1984) CT of extracranial hemorrhage and hematomas. Am J Roentgenol 143:907-912. https:// doi.org/10.2214/ajr.143.4.907

10. Specchi S, Auriemma E, Morabito S, Ferri F, Zini E, Piola V, Pey P, Rossi F (2017) Evaluation of the computed tomographic "sentinel clot sign" to identify bleeding abdominal organs in dogs with hemoabdomen. Vet Radiol Ultrasound 58:18-22. https://doi.org/ 10.1111/vru. 12439

11. Ampanozi G, Halbheer D, Ebert LC, Thali MJ, Held U (2020) Postmortem imaging findings and cause of death determination compared with autopsy: a systematic review of diagnostic test accuracy and meta-analysis. Int J Legal Med 134:321-337

12. Chevallier C, Doenz F, Vaucher P et al (2013) Postmortem computed tomography angiography vs. conventional autopsy: advantages and inconveniences of each method. Int J Legal Med 127:981-989

13. Reijnders UJ, Berger FH, Giannakopoulos GF et al (2015) Postmortem imaging compared with autopsy in trauma victims - a systematic review. Forensic Sci Int 257:29-48. https://doi.org/10. 1016/j.forsciint.2015.07.026

14. Christe A, Flach P, Ross S, Spendlove D, Bolliger S, Vock P, Thali MJ (2010) Clinical radiology and postmortem imaging (Virtopsy) are not the same: specific and unspecific postmortem signs. Legal Med 12:215-222. https://doi.org/10.1016/j.legalmed.2010.05.005

15. Carballeira Álvarez A, Mancini J, Tuchtan-Torrents L, Gach P, Bartoli C, Desfeux J, Piercecchi MD, Gorincour G (2018) Diagnostic value of unenhanced postmortem computed tomography in the detection of traumatic abdominal injuries. Diagn Interv Imaging 99:397-402. https://doi.org/10.1016/j.diii.2017.12.015

16. Christe A, Ross S, Oesterhelweg L, Spendlove D, Bolliger S, Vock P, Thali MJ (2009) Abdominal trauma-sensitivity and specificity of postmortem noncontrast imaging findings compared with autopsy findings. J Trauma - Inj Infect Crit Care 66:1302-1307. https://doi. org/10.1097/TA.0b013e31818c1441

17. O’Donnell C, Bedford P, Burke M (2011) Massive hemoperitoneum due to ruptured ectopic gestation: postmortem CT findings in a deeply frozen deceased person. Legal Med 13: 245-249. https://doi.org/10.1016/j.legalmed.2011.05.006

18. Burke M, O'Donnell C (2015) Postmortem computed tomography findings in ruptured splenic artery aneurysm. Am J Forensic Med Pathol 36:224-226. https://doi.org/10.1097/paf.0000000000000180

19. Jackowski C, Thali M, Aghayev E, Yen K, Sonnenschein M, Zwygart K, Dirnhofer R, Vock P (2006) Postmortem imaging of blood and its characteristics using MSCT and MRI. Int J Legal Med 120:233-240. https://doi.org/10.1007/s00414-005-0023-4

20. Madea B (2015) Rechtsmedizin, 3rd edn. Springer, Berlin Heidelberg

21. Brinkmann B, Madea B (2004) Handbuch gerichtliche Medizin. Springer, Berlin Heidelberg

22. Zech WD, Jackowski C, Buetikofer Y, Kara L (2014) Characterization and differentiation of body fluids, putrefaction fluid, and blood using Hounsfield unit in postmortem CT. Int J Legal Med 128:795-802

23. Saukko P, Knight B (2016) Knight's forensic pathology. In: 4th ed. Edward Arnold: London UK, pp 79-90

24. DiMaio VJ, D D (2001) Forensic pathology. In: 2nd ed. CRC Press. Boca Raton, London, New York, Washington D.C., pg. 21

25. Flach PM, Gascho D, Schweitzer W, Ruder TD, Berger N, Ross SG, Thali MJ, Ampanozi G (2014) Imaging in forensic radiology: an illustrated guide for postmortem computed tomography technique and protocols. Forensic Sci Med Pathol 10:583-606. https://doi.org/10.1007/s12024-014-9555-6

26. Norzailin AB, Noor Azman S, Mohd Helmee MN, Khairul Anuar Z (2016) The sensitivity, specificity and predictive values of post mortem computed tomography in detecting liver and splenic injury due to road traffic accident. Med J Malaysia 71(1):1-7

27. Juźwik E, Moskała A, Woźniak K et al (2019) Evaluation of usefulness of post-mortem computed tomography in the diagnosis of abdominal parenchymal organ injuries compared to medicolegal autopsy findings. Arch Med Sadowej Kryminol 69:40-55

28. Watanabe S, Hyodoh H, Shimizu J, Okazaki S, Mizuo K, Rokukawa M (2015) Classification of hemopericardium on postmortem CT. Legal Med 17:376-380. https://doi.org/10.1016/j. legalmed.2015.05.004

29. Yamaguchi R, Makino Y, Chiba F, Torimitsu S, Yajima D, Shinozaki T, Iwase H (2015) Fluid-fluid level and pericardial hyperdense ring appearance findings on unenhanced postmortem ct can differentiate between postmortem and antemortem pericardial hemorrhage. Am J Roentgenol 205:W568-W577. https://doi. org/10.2214/AJR.15.14808

30. Coffin A, Boulay-Coletta I, Sebbag-Sfez D, Zins M (2015) Radioanatomy of the retroperitoneal space. Diagn Interv Imaging 96:171-186. https://doi.org/10.1016/j.diii.2014.06.015

Publisher's note Springer Nature remains neutral with regard to jurisdictional claims in published maps and institutional affiliations. 